\title{
Improvement Harmony Search Algorithm and Its Application
}

\author{
Hongtao $\mathrm{Yu}^{1, \mathrm{a}}$, Shangzhen Zhu, ${ }^{1, b}$, Yanhua Lei ${ }^{1, \mathrm{c}}$ and Xinli Chi ${ }^{1, \mathrm{~d}}$ \\ ${ }^{1}$ College of Automation, Shenyang Institute of Engineering, Shenyang Liaoning110136, China \\ aneu970773@sohu.com, bhusz@sie.edu.cn, 'Leiyh@si.edu.cn, dChixl@si.edu.cn,
}

\section{Keywords: Harmony search algorithm; Exploration; Exploitation; PID control; Entropy}

\begin{abstract}
To solve the problem that harmony search (HS) algorithm is easily trapped into local optimal, a novel improved harmony search (IHS) algorithm is proposed. Search strategy are redefined in IHS. In order to increase the diversity of harmony in harmony memory and enhance exploration, the concept of entropy is introduced for generating new harmony at the beginning of the optimization. When the algorithm is at a standstill state during the process of optimization, the information of harmony memory is considered and the basic theory of firefly algorithm is introduced so as to accelerate the convergence speed of the algorithm and improve the algorithm's intensification ability. The proposed algorithm is tested on several classic functions and PID control problem, and the simulation results show that IHS has better performance.
\end{abstract}

\section{Introduction}

Harmony search (HS) algorithm is a novel meta heuristic algorithm proposed by Geem et al. [1] in 2001. In order to make up for the above shortcomings and improve the quality of the algorithm, scholars have put forward many improved harmony search algorithms. To solve the problem of discrete variables, a variety of harmony search algorithm was presented by Geem [2]. This algorithm could ensure the diversity of the solution vector by restricting the same number of harmonics in the harmony memory. Wang et al. [3] proposed an adaptive harmony search algorithm (SAHS) based on the adaptive adjustment of the position information of the harmony memory database. Chen et al. [4] presented a harmony search algorithm with dynamic control parameters, which was the dynamic adjustment of the parameters and the step parameter (NDHS). Yadav et al. [5] improved the strategy of gene regulation, and proposed an intelligent control harmony search algorithm based on harmony memory bank (ITHS). Wang et al. [6] proposed a harmony search algorithm based on differential evolution algorithm (CDEHS).

This paper presents a new improved harmony search (IHS) algorithm based on the information of the harmony memory, in which the feedback mechanism is formed and the optimization strategy of the harmony search algorithm is changed. In this process, the effectiveness of the introduction of external information and the internal information sharing of the harmony memory are considered.

\section{Improved Harmony Search (IHS) Algorithm}

Harmony Search (HS) Algorithm. HS algorithm is a simulation of the control process of music in tones of musical performers. In music improvisation process, musical performers repeatedly adjust the tone of the instruments so as to achieve a pleasing harmony. Musical performers seek to find pleasing harmony as determined by aesthetic standard, just as the optimization process seeks to find global optimization solution [7]. The pitch of each musical instrument determines the aesthetic quality, just as the objective function value is determined by the set of values assigned to each decision variable.

The IHS algorithm is proposed in this paper based on the definition of the population diversity and stagnation state.

Exploration Strategy. Exploration strategy aims to improve exploration ability. The better exploration ability expresses that the ability of the individual to search the unknown region is stronger 
in the global scope, that is, the global search ability is stronger. In order to describe the search strategy, the following definition is proposed in this paper.

Definition 1: Decision variables entropy

If $\mathrm{m}$ is the number of solution vector, and $\mathrm{n}$ is the number of decision variables, the entropy $H_{j}$ for decision variables $j$ can be defined as follows[8]:

$$
\begin{aligned}
& H_{j}=\underbrace{\text { 邋 }_{k=i+1}^{m}\left(-p_{i k} \log p_{i k}\right)}_{i=1} \\
& p_{i k}=1-\frac{\left|x_{j}^{i}-x_{j}^{k}\right|}{A_{j}^{\max }-A_{j}^{\min }}
\end{aligned}
$$

Where $A_{j}^{\max }$ and $A_{j}^{\min }$ are the maximum and minimum values for decision variables $j$, and $p_{i k}$ is probability that decision variables $j$ for solution vector $i$ is different from decision variables $j$ for solution vector $k$.

In exploration stage for the proposed algorithm, a new harmony is not generated on account of random selection, memory consideration and pitch adjustment, but it is improvised only by random selection and memory consideration. In order to improve the diversity of the population, enhance the exploration ability, decision variables entropy based on random selection must exceed a certain value.

Exploitation Strategy. Exploitation strategy aims to improve exploitation ability. The better exploitation ability represents that the ability of the individual to search local region is stronger, that is, the local search ability is stronger. In order to describe the search strategy, the definition is proposed as follows.

Definition 2: The harmony memory entropy

Entropy $\mathrm{H}$ for group is got by averaging decision variables entropy $H_{j}$

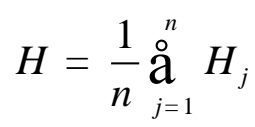

Definition 3: Standstill

If the entropy for the harmony memory doesn't change after finite iterations, HS algorithm is at a standstill.

When the algorithm is in a state of stagnation, the objective function value of harmony vectors based on the exploration strategy is better than the objective function value of the worst harmony in harmony memory hardly. At this time, the harmony vectors in the harmony memory will not be updated, and it will only increase the search time in vain, so the search strategy must be changed. In this paper, the optimal solution is further exploited by using the information of current harmony vector in the harmony memory, which mainly involves in the idea of artificial firefly algorithm. Each harmony vector of the harmony memory is regarded as a firefly. The movement is decided by fluorescent brightness of each firefly, and the movement distance of the firefly is determined by the attraction of the firefly. Related definitions [9] are as follows:

Definition 4: Fluorescent brightness of firefly

$$
I=I_{0} e^{-g r_{i j}}
$$

Where $r_{i j}$ is the distance between firefly $i$ and firefly $j, g$ is light absorption coefficient, which shows the characteristic that the fluorescence decreases with the increase of distance. $I_{0}$ is its fluorescent brightness, is also the largest fluorescence brightness, which is related to the value of the objective function. The maximum fluorescence brightness is proportional to the objective function value.

Definition 5: The attraction of firefly 
$b=b_{0} e^{-g r_{i j}^{2}}$

Where $b_{0}$ is the largest attraction, the meaning of $g$ and $r_{i j}$ are identical with the above.

Definition 6: Location update

$x_{i}=x_{i}+b\left(x_{j}-x_{i}\right)$

\section{The Step of IHS Algorithm}

The HIS algorithm works in this paper as follow:

Step 1: initialize the problem and algorithm parameters

In this step, the optimization problem is specified as follows:

$f(x) ; x_{i}$ ? $X_{i}(i \quad 1,2, \mathrm{~L}, N)$

Where $f(x)$ is an objective function; $x$ is a solution vector composed of decision variable $x_{i} ; N$ is the number of decision variables; $X_{i}$ is the set of the possible range of values for each decision variable; $n$ is the number of attempt; limit is the limit number. In addition, the harmony memory size (HMS) and the maximum number of iterations (NI) are given in this step.

Step 2: initialize the harmony memory

In this step, the harmony memory matrix is filled with as many randomly generated solution vectors as the HM.

Step 3: generation of a new harmony

A new harmony $X^{\prime}=\left(x_{1}^{\prime}, x_{2}^{\prime}, \mathrm{L}, x_{n}^{\prime}\right)$ is improvised based on the following search strategy.

1) Exploration strategy

The value of decision variables $x_{i}^{\prime}$ can be randomly chosen from the possible value range, but the decision variables entropy must subject to constraint, which can be described as follows:

$$
\begin{aligned}
& x_{j}^{\prime} \hat{\mathrm{I}} X_{j} \\
& H_{j}>H_{0}
\end{aligned}
$$

If the decision variables satisfy conditions, it will be received. Otherwise, it will be rejected. The decision variables will be generated based on memory consideration after the number of attempt is $n$.

If the harmony memory entropy does not change when number of iteration is over limit, exploitation strategy will be carried out.

2) Exploitation strategy

Objective function value of each harmony vector is regarded as the maximum fluorescence brightness of each firefly.

Fluorescent brightness and attraction can be got according to Eq.4 and Eq.5, and Location can be updated based on Eq.6.

Step 4: update the harmony memory

The new harmony vector replaces the worst harmony in the HM, if its objective function value is better than that of the worst harmony.

Step 5: check stopping criterion

Steps 3 and 4 are repeated until the termination criterion (NI) is satisfied.

\section{Results of Numerical Simulation}

Selection of Test Functions. In order to test the performance of the proposed algorithm, it is applied to deal with three benchmark functions. In addition, the results of this algorithm are compared with Refs. [1, 3-6]. The results are shown in Table 1 in the 20 independent runs by each algorithm. 
Where $\mathrm{HMS}=5, \mathrm{n}=3$, limit=300, NI=50 000, and the other parameters are the same to relevant reference.

Table 1 The optimization results of $f_{1} \sim f_{3}$

\begin{tabular}{cccccc}
\hline$f$ & algorithm & best & worst & mean & SD \\
\hline Sphere & HS & $6.9231 \mathrm{e}-001$ & $4.2771 \mathrm{e}+000$ & $8.1284 \mathrm{e}-001$ & $7.5724 \mathrm{e}-001$ \\
& SAHS & $5.1832 \mathrm{e}-002$ & $5.2574 \mathrm{e}-001$ & $1.3785 \mathrm{e}-001$ & $4.3746 \mathrm{e}-001$ \\
& DNHS & $6.2147 \mathrm{e}-003$ & $4.5241 \mathrm{e}-001$ & $0.5274 \mathrm{e}-001$ & $4.5874 \mathrm{e}-002$ \\
& ITHS & $4.1825 \mathrm{e}-004$ & $4.5241 \mathrm{e}-002$ & $1.4582 \mathrm{e}-002$ & $2.7541 \mathrm{e}-002$ \\
& CDEHS & $4.2879 \mathrm{e}-002$ & $4.8574 \mathrm{e}-001$ & $0.9874 \mathrm{e}-001$ & $3.9874 \mathrm{e}-001$ \\
& IHS & $5.2874 \mathrm{e}-005$ & $5.1285 \mathrm{e}-002$ & $1.5879 \mathrm{e}-002$ & $1.4771 \mathrm{e}-002$ \\
Griewank & HS & $2.2657 \mathrm{e}+001$ & $5.2678 \mathrm{e}+001$ & $3.7985 \mathrm{e}+001$ & $6.9874 \mathrm{e}+000$ \\
& SAHS & $1.4874 \mathrm{e}+000$ & $2.4178 \mathrm{e}+000$ & $1.7874 \mathrm{e}+000$ & $2.0174 \mathrm{e}-001$ \\
& DNHS & $3.0523 \mathrm{e}-001$ & $2.2356 \mathrm{e}+000$ & $1.2583 \mathrm{e}+000$ & $4.5327 \mathrm{e}-001$ \\
& ITHS & $1.3172 \mathrm{e}-002$ & $1.1253 \mathrm{e}+000$ & $4.7815 \mathrm{e}-001$ & $3.7784 \mathrm{e}-001$ \\
& CDEHS & $1.2414 \mathrm{e}+000$ & $2.2642 \mathrm{e}+000$ & $1.6936 \mathrm{e}+000$ & $1.8725 \mathrm{e}-001$ \\
& IHS & $4.2574 \mathrm{e}-003$ & $3.3478 \mathrm{e}-001$ & $1.5963 \mathrm{e}-002$ & $8.8516 \mathrm{e}-002$ \\
Rastrigin & HS & $2.5662 \mathrm{e}-002$ & $3.1256 \mathrm{e}+000$ & $8.4127 \mathrm{e}-001$ & $4.3818 \mathrm{e}+000$ \\
& SAHS & $2.1542 \mathrm{e}-002$ & $1.5236 \mathrm{e}+000$ & $4.7895 \mathrm{e}-001$ & $3.2875 \mathrm{e}+000$ \\
& DNHS & $5.2347 \mathrm{e}-003$ & $1.65247+000$ & $2.3542 \mathrm{e}-001$ & $3.3552 \mathrm{e}+000$ \\
& ITHS & $2.3145 \mathrm{e}-006$ & $1.1485 \mathrm{e}+000$ & $0.5144 \mathrm{e}-001$ & $1.5670 \mathrm{e}+000$ \\
& CDEHS & $2.0278 \mathrm{e}-002$ & $1.3125 \mathrm{e}+000$ & $4.6936 \mathrm{e}-001$ & $2.9547 \mathrm{e}+000$ \\
& IHS & $1.9865 \mathrm{e}-006$ & $0.7963 \mathrm{e}-001$ & $2.5508 \mathrm{e}-002$ & $1.2978 \mathrm{e}+000$ \\
\hline
\end{tabular}

As can be seen from the Table 1, the IHS algorithm can balance the exploration and exploitation ability.

\section{Experimental Results and Analysis for PID Control Problem}

In order to further verify the performance of the IHS algorithm, the PID control problem [10] is dealt with it, and the 20 independent runs are carried out. Taking into account the comparable performance with the literature [10], the IHS algorithm parameters used are HMS $=5, \mathrm{NI}=1000$ and limit=50. The PID control problem model can see literature [10].

Example: $d_{1}=d_{2}=0.36, e_{1}=e_{2}=1, m=10, n u m=60$, 当 $k=-m,-m+1, \mathrm{~L} 0$ 时, $x(k)=0.5, y(k)=-0.5$

The results of Example got by IHS are $K_{p}=0.6494, K_{i}=0.1695, K_{d}=0.2732$ and $J=2.6937$. The objective function value is 2.6937 , which is less than 3.6937 that is the objective function value of literature [11]. The evolution curve of the average function value for example 1 is shown in Fig. 1.

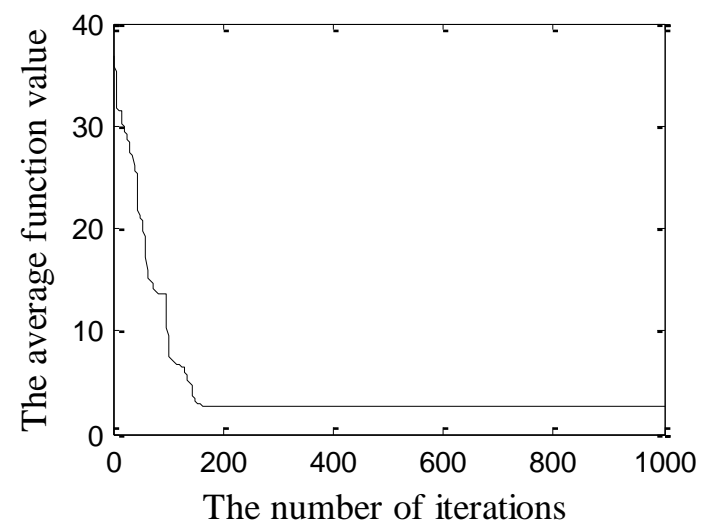

Figure 1. Evolution curve of average value function 
As can be seen from Fig. 1, the IHS algorithm proposed in this paper not only has a better solution quality, but also has a faster convergence speed.

\section{Summary}

The HS algorithm is easy to fall into local optimum, and its convergence accuracy is not high. This paper presents a new IHS algorithm, which can balance the exploration and exploitation ability. The IHS uses the concept of entropy to produce new harmony, which can increase the diversity of the harmony memory and improve the global search ability of the algorithm. In addition, when the algorithm falls into local optimum, the IHS defines the exploitation strategy. In this way, the local search is performed based on the information of the harmony memory. From the experimental results of the standard test function and the PID control problem, we can see that the IHS algorithm proposed in this paper improves the search accuracy and stability of the HS algorithm.

\section{Acknowledgements}

This work was supported in part by the Liaoning Province Dr. started fund under Grant 201501087 and Science Research General Project of Liaoning Education Department under Grant L2014530, L2014517, L2015367 and L2015366.

\section{References}

[1] Z.W. Geem, J.H. Kim and G.V. Loganathan. A new heuristic optimization algorithm: harmony search [J], Simulation, Vol. 76 (2001) No.2, p. 60.

[2] Z.W. Geem. Effects of initial memory and identical harmony in global optimization using harmony search algorithm [J]. Applied Mathematics and Compulation, Vol. 218 (2012) No.22, p. 11337.

[3] C.M. Wang and Y.F. Huang. Self-adaptive harmony search algorithm for optimization [J].Expert Systems with Applications, Vol. 37 (2010) No.4, p. 2826.

[4] J. Chen, Q.K. Pan and J.Q. Li. Harmony search algorithm with dynamic control parameters [J]. Applied Mathematics and Computation, Vol. 219(2012) No.2, p. 592.

[5] P. Yadav, R. Kumar, S. Panda and C.S. Chang. An Intelligent tuned harmony search algorithm for optimization [J]. Information Sciences, Vol. 196 (2012) No.2, p. 47.

[6] L. Wang and L.P. Li. A coevolutionary differential evolution with harmony search for reliabilityredundancy optimization [J]. Expert Systems with Applications, Vol. 39 (2012) No.5, p. 5271.

[7] A. Verma, B.K. Panigrahi and P.R. Bijwe. Harmony search algorithm for transmission network expansion planning [J], Generation, Transmission \& Distribution, IET, Vol. 4 (2010) No.6, p. 663.

[8] X.H. Yuan, Y.B. Yuan and C. Wang. A novel self-adaptive chaotic genetic algorithm [J], Acta Electronica Sinica, Vol. 34 (2006) No.4, p. 708. (In Chinese)

[9] H.T. Yu, L.Q. Gao and X.C. Han. Discrete artificial firefly algorithm for solving traveling salesman problem [J]. Journal of South China University of Technology (Natural Science Edition), Vol. 43 (2015) No.1, p. 126. (In Chinese)

[10] [D.X. Zou, X. Wang and N. Duan. An Improved Particle Swarm Optimization Algorithm for Chaotic Synchronization Based on PID Control [J]. Journal of Information \& Computational Science, Vol. 11 (2014) No.9, p. 3177. 